Received: 23.04 .2021

Revised: 22.06 .2021

Accepted: 25.06 .2021

DOI: $10.17804 / 2410-9908.2021 .3 .031-044$

\title{
NEUTRON STUDIES OF THE V-4Ti-4Cr ALLOY
}

\author{
S. G. Bogdanov ${ }^{1, a^{*}}$, I. F. Berger ${ }^{1, \text { b) }}$, V. I. Bobrovskii ${ }^{1, c)}$, V. I. Voronin ${ }^{1, \text { d) }}$, \\ V. D. Parkhomenko, ${ }^{1, \text { e) }}$, and V. M. Chernov ${ }^{2, \text { f) }}$ \\ ${ }^{1}$ M. N. Miheev Institute of Metal Physics, Ural Branch of the Russian Academy of Sciences, \\ 18 S. Kovalevskoy St., Ekaterinburg, 620108, Russian Federation \\ ${ }^{2}$ A. A. Bochvar High-Technology Scientific Research Institute for Inorganic Materials (VNIINM JSC), \\ 5a Rogova St., Moscow, Russian Federation
}


4. Voronin V.I. Neutronography of Irradiated Reactor Austenitic Steels. Crystallography Reports, 2021, vol. 66, No. 2, pp. 314-322. DOI: 10.1134/S1063774521020127.

5. Voronin V.I., Arbuzov V.L., Bobrovskii V.I., Danilov S.E., Kozlov K.A., Proskurnina N.V., Sagaradze V.V. Peculiarities of radiation-induced processes in the Cr-Ni-Mo austenitic steels studied by neutron diffraction. Diagnostics, Resource and Mechanics of materials and structures, 2015, iss. 5, pp. 80-89. DOI: 10.17804/2410-9908.2015.5.080-089. Available at: DREAM_Issue_5_2015_ _Voronin V.I. et al._080_089.docx

6. Rouxel B., Bisor C., De Carlan Y., Courcelle A., Legris A. Influence of the austenitic stainless steel microstructure on the void swelling under ion irradiation. EPJ Nuclear Sci. Technol., 2016, vol. 2, pp. 30. DOI: 10.1051/epjn/2016023.

7. Arbuzov V.L., Goshchitskii B.N., Sagaradze V.V., Danilov S.E., Zuev Yu.N., Kar'kin A.E., Parkhomenko V.D., Kozlov A.V., Chernov V.M. The accumulation and annealing of radiationinduced defects and effect of hydrogen on the physicomechanical properties of the $\mathrm{V}-4 \mathrm{Ti}-4 \mathrm{Cr}$ vanadium-based alloys under low-temperature (at $77 \mathrm{~K}$ ) neutron irradiation. Physics of Metals and Metallography, 2016, vol. 117, No. 3, pp. 299-306. DOI: 10.1134/S0031918X16030030.

8. Arbuzov V.L., Goschitskiy B.N., Danilov S.E., Sagaradze V.V., Antonova O.V., Raspopova G.A., Chernov V.M., Kozlov A.V. Microstructure of V-4Ti-4Cr alloy, irradiated by electrons, neutrons and ions of deuterium after various heat treatments. Voprosy atomnoy nauki i tekhniki (VANT). Series Material science and new materials, 2018, No. 3 (94), P. 4-12. (In Russian). Available at: http://www.bochvar.ru/vopros/

9. Blokhin D.A., Leontyeva-Smirnova M.V., Chernov V.M., Blokhin A.I., Demin N.A., Sipachev I.V. Nuclear Physical Properties of Ferritic-Martensitic Steel EK-181 under Conditions of Long Term Neutron Irradiation in Fast Breeder BN-600 and Fusion DEMO-RF Reactors. Inorganic Materials: Applied Research, 2011, vol. 2, No. 2, pp. 129-135. DOI: 10.1134/S2075113311020067.

10. Chernov V.M., Leont'eva-Smirnova M.V., Potapenko M.M., Polekhina N.A., Litovchenko I.Yu., Tyumentsev A.N., Astafurova E.G., and Khromova L.P. Structure-Phase Transformations and Physical Properties of Ferritic-Martensitic $12 \%$ Chromium Steels EK-181 and ChS-139. Technical Physics, 2016, vol. 61, No. 1, pp. 97-102. DOI: 10.1134/S1063784216010084.

11. Rodriguez-Carvajal J. Recent advances in magnetic structure determination by neutron powder diffraction. Physica B, 1993, vol.192, pp. 55-64. DOI: 10.1016/0921-4526(93)90108-I.

12. Ungar T., Borbely A. The effect of dislocation contrast on X-ray line broadening: a new approach to line profile analysis. Appl. Phys. Lett., 1996, vol. 69 (21), pp. 3173-3175. DOI: 10.1063/1.117951.

13. Balogh L., Brown D.W., Mosbrucker P., Long F., Daymond M.R. Dislocation structure evolution induced by irradiation and plastic deformation in the $\mathrm{Zr}-2.5 \mathrm{Nb}$ nuclear structural material determined by neutron diffraction line profile analysis. Acta Materialia, 2012, vol. 60, pp. 5567-5577. DOI:10.1016/j.actamat.2012.06.062.

14. Bale H.D., Schmidt P.W. Small angle X-ray scattering investigation of submicroscopic porosity with fractal properties. Phys. Rev. Lett., 1984, vol. 53, pp. 596-604. DOI: 10.1103/physrevlett.53.596.

15. Debye P., Anderson H.R., Brumberger H. Scattering by an inhomogeneous solid. II. The correlation function and its application. J. Apll. Phys., 1957, vol. 28, pp. 679-684.

16. Bogdanov S.G., Goshchitskii B.N., Parkhomenko V.D., Leontieva-Smirnova M.V., Chernov V.M. Small-Angle Neutron Scattering Investigation of the Nanostructure of FerriticMartensitic $12 \%$-Chromium Steels. Physics of Solid State, 2014, vol. 56, No. 1, pp. 1-13. DOI: $10.1134 / \mathrm{S} 1063783414010065$.

17. Bogdanov S.G., Goshchitskii B.N., Parkhomenko V.D. Small-Angle Neutron Scattering Investigation of the Substructure of Nickel Irradiated with Fast Neutrons. Physics of Metals and Metallography, 2014, vol. 115, No. 6, pp. 566-569. DOI: 10.1134/S0031918X14060039.

18. Feigin L.A., Svergun D.I. Structure Analysis by Small-Angle X-Ray and Neutron Scattering, Plenum Press, New York, 1987, 335 p. ISBN 0-306-42629-3. 
Подана в журнал: 23.04.2021

УДК 620.186:546.11: 669.15-194.56:539.12.043: 521.039.546.3:669.14

DOI: $10.17804 / 2410-9908.2021 .3 .031-044$

\title{
НЕЙТРОНОГРАФИЧЕСКИЕ ИССЛЕДОВАНИЯ СПЛАВА V-4Ti-4Cr
}

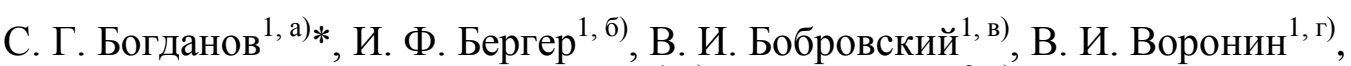 \\ В. Д. Пархоменко ${ }^{1, \text { д) }}$, В. М. Чернов ${ }^{2, \text { e) }}$ \\ ${ }^{1}$ Институт физики металлов Уральского отделения Российской академии наук, имени М.Н.Михеева, \\ д.18, ул. С. Ковалевской, г. Екатеринбург, Российская Федераџия \\ ${ }^{2}$ АО «Высокотехнологический научно-исследовательский институт \\ неорганических материалов имени академика А.А. Бочвара», \\ д. 5а, ул. Рогова, г. Москва, Российская Федераџия \\ a) (ID https://orcid.org/0000-0003-2327-1180 @ bsg@imp.uran.ru;

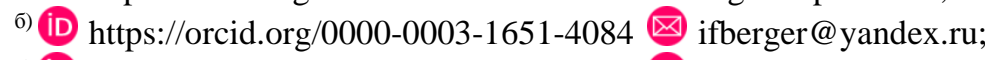 \\ в) (ID https://orcid.org/0000-0002-4692-8889 bobrovskii@imp.uran.ru;

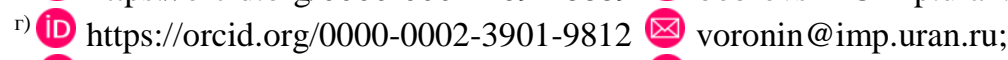 \\ д) (ID https://orcid.org/0000-0002-4332-8992@ parkhomenko@imp.uran.ru; \\ e) (iD https://orcid.org/0000-0002-2049-5667 @ VMChernov@ @ochvar.ru \\ *Ответственный автор. Электронная почта: bsg@imp.uran.ru \\ Адрес для переписки: ул. С. Ковалевской, 18, г. Екатеринбург, Российская Федерация \\ Тел.: +7 (343) 374-00-03; факс: +7 (343) 374-00-03
}

Достижение конкурентных экономических показателей энергетических реакторов на быстрых нейтронах требует обеспечения высоких коэффициентов выгорания топлива, для чего необходимо существенное повышения радиационной стойкости оболочек топливных элементов. В качестве перспективных оболочечных материалов в настоящее время предлагаются малоактивируемые ванадиевые сплавы, обладающие повышенной радиационной и термической стойкостью в широких интервалах температур и доз радиационной повреждаемости. Методами нейтронной дифракции и малоуглового рассеяния нейтронов исследована микроструктура сплава V-4Ti-4Cr, облученного быстрыми нейтронами в диапазоне флюенсов до $1 \cdot 10^{20} \mathrm{~cm}^{-2}$. Определены характеристики минорных преципитатных фаз в системе. Показана возможность и перспективность использования нейтронографических методов для изучения радиационного поведения этой категории сплавов.

Ключевые слова: реакторы на быстрых нейтронах, радиационные повреждения, оболочечные материалы, ванадиевые сплавы, нейтронная дифракция, малоугловое рассеяние нейтронов.

\section{1. Введение}

Решение проблемы достижения высоких экономических показателей реакторов на быстрых нейтронах связано с обеспечением высоких коэффициентов выгорания топлива, т. е. возможности максимально длительной безопасной эксплуатации топливных сборок [1]. Это требует создания материалов оболочек тепловыделяющих элементов (ТВЭЛов), способных работать в условиях значительно более высоких температур, чем в традиционных водяных реакторах, в контакте с жидкометаллическими теплоносителем и при высокодозовом облучении быстрыми нейтронами. К счастью, набор химических элементов, пригодных для использования в оболочечных сплавах для быстрых реакторов, гораздо шире, чем для реакторов на медленных нейтронах вследствие значительно меньших величин сечений захвата быстрых нейтронов по сравнению с медленными. Это дает возможность применять в качестве оболочечных материалов быстрых реакторов сложные многокомпонентные стали и 
сплавы [2]. В реакторах на медленных нейтронах такое меню фактически исчерпывается цирконием и алюминием.

В настоящее время в качестве оболочечных материалов ТВЭЛов реакторов на быстрых нейтронах наиболее широко используются аустенитные высоконикелевые стали [3-6]. Эти стали обладают высокой прочностью, жаростойкостью, без проблем выдерживают контакт с жидким натрием и, вдобавок, они высокотехнологичны. Однако при достижении высоких доз нейтронного облучения (выше $70 \mathrm{CHA} \mathrm{-} \mathrm{смещений} \mathrm{на} \mathrm{атом)} \mathrm{в} \mathrm{них} \mathrm{развивается} \mathrm{ва-}$ кансионное распухание, ограничивающее возможность эксплуатации изделия пределом порядка (90-100) СНА. В настоящее время в мире предпринимаются интенсивные попытки поднять этот предел. Требуемый экономикой показатель составляет 150 СНА.

В качестве альтернативных оболочечных материалов предлагаются малоактивируемые ванадиевые сплавы (основной сплав V-4Ti-4Cr), обладающие повышенной радиационной и термической стойкостью в широких интервалах температур и доз радиационной повреждаемости $[7,8]$. Однако особенностью сплавов ванадия является появление в них в процессе производства газовых примесей внедрения (кислород, водород, азот, углерод и т. д.), существенно сказывающихся на их физико-механических свойствах.

Рассматриваются также возможность использования некоторых ферритно-мартенситных сталей $[9,10]$. Они хорошо противостоят вакансионному распуханию, но зато подвержены радиационному охрупчиванию, угрожающему целостности оболочек ТВЭЛов.

Свойства всех этих систем помимо многокомпонентного состава определяются их микроструктурой - наличием преципитатов минорных фаз, зеренной структурой, текстурой, сеткой дислокаций, созданной в процессе изготовления изделий и эволюционирующей в результате радиационно-индуцированных процессов при их эксплуатации. Осознанное улучшение свойств таких материалов требует понимания механизмов этих процессов, которое может быть достигнуто лишь при комплексном, мультимасштабном их исследовании.

Нашей задачей было изучение возможностей использования нейтронографических методов для исследования радиационно-индуцированных явлений в облученных быстрыми нейтронами оболочечных сплавах на основе ванадия.

\section{2. Материал и методика}

Образцы сплава V-4Ti-4Cr, предварительно подвергнутые 30 \% «холодной» деформации и термообработкам ТО-1 и ТО-2 и облучению до различных доз нейтронами при температуре $80{ }^{\circ} \mathrm{C}$ в реакторе ИВВ-2М, исследовались методами нейтронного структурного анализа и малоуглового рассеяния нейтронов на приборах, установленных на горизонтальных каналах этого же реактора.

Для дифракционных исследований использовался нейтронный дифрактометр высокого разрешения Д7а. Длина волны монохроматических нейтронов $\lambda=1,5302 \AA$, угловое разрешение дифрактометра составляло $\Delta \mathrm{d} / \mathrm{d}=0,002$.

Малоугловые измерения выполнены на дифрактометре малоуглового рассеяния на поляризованных нейтронах Д6. Диапазон углов, доступный для измерения на дифрактометре, составлял около $5^{\circ}$, средняя длина волны 0,5 нм, разрешение по длинам волн $30 \%$. Погрешность определения углового положения детектора не превышала \pm 2 мин. Погрешность определения плотности потока нейтронного излучения не более $\pm 0,6 \%$. Погрешность измерения интегральной интенсивности нейтронов на контрольном образце не выше $\pm 0,8 \%$.

Образцы из сплава V-4Ti-4Cr, представляющего собой твердый ОЦК раствор ванадия, хрома и титана, в объеме которого, как показали данные дальнейших исследований, содержатся преципитаты минорных фаз, были выполнены в виде цилиндров диаметром 6 мм и длиной 40 мм и имели химический состав: 
Таблица 1 - Химический состав образцов сплава V-4Ti-4Cr (мас., \%)

\begin{tabular}{|c|c|c|c|c|c|c|c|c|c|}
\hline $\mathrm{V}$ & $\mathrm{Ti}$ & $\mathrm{Cr}$ & $\mathrm{Fe}$ & $\mathrm{C}$ & $\mathrm{O}$ & $\mathrm{N}$ & $\mathrm{Al}$ & $\mathrm{Si}$ & $\mathrm{Cu}$ \\
\hline 91,845 & 4,0 & 4,0 & 0,025 & 0,02 & 0,04 & 0,015 & 0,02 & 0,025 & 0,01 \\
\hline
\end{tabular}

Режимы термообработок образцов (вакуум - $10^{-5}$ мм. рт. ст.; охлаждение с печью):

TO-1: $\left(350^{\circ} \mathrm{C} / 1\right.$ ч $) \rightarrow\left(550{ }^{\circ} \mathrm{C} / 1 \mathrm{ч}\right) \rightarrow\left(650^{\circ} \mathrm{C} / 40 \mathrm{мин}\right) \rightarrow\left(1080^{\circ} \mathrm{C} / 1 \mathrm{ч}\right)$

TO-2: $\left(350^{\circ} \mathrm{C} / 1\right.$ ч) $\rightarrow\left(550{ }^{\circ} \mathrm{C} / 1\right.$ ч $) \rightarrow\left(650^{\circ} \mathrm{C} / 40\right.$ мин $) \rightarrow\left(1080^{\circ} \mathrm{C} / 3\right.$ ч $)$

\section{3. Результаты и обсуждение}

Необходимо отметить, что некогерентно рассеивающий ванадий и его сплавы являются весьма трудными объектами для нейтронно-дифракционных исследований. Система V-4Ti-4Cr в этом смысле не исключение. Более того, из-за разных знаков амплитуд когерентного рассеяния $\left(b_{\mathrm{V}}=-0,382 \Phi_{\mathrm{M}} ; b_{\mathrm{Cr}}=3,635 \Phi_{\mathrm{M}} ; b_{\mathrm{Ti}}=-3,438\right.$ Фм) среднее значение амплитуды рассеяния атомов получается равным $-0,35$ Фм, т. е. твердый раствор представляет собой почти нулевую матрицу. Это факт затрудняет получение качественного дифракционного спектра, но зато способствует выявлению преципитатов. В табл. 2 дана подборка ядерно-физических характеристик составляющих сплав элементов.

Таблица 2 - Амплитуда ядерного рассеяния элементов $b$ и состав образцов сплава V-4Ti-4Cr в массовых и атомных процентах

\begin{tabular}{|c|c|c|c|}
\hline Элемент & $b, \Phi_{\mathrm{M}}\left(10^{-13} \mathrm{cм}\right)$ & Мас. \% & Ат. \% \\
\hline $\mathrm{V}$ & $-0,382$ & 91,845 & 91,479 \\
\hline $\mathrm{Ti}$ & $-3,438$ & 4 & 4,239 \\
\hline $\mathrm{Cr}$ & 3,635 & 4 & 3,903 \\
\hline $\mathrm{Fe}$ & 9,45 & 0,025 & 0,023 \\
\hline $\mathrm{C}$ & 6,648 & 0,02 & 0,084 \\
\hline $\mathrm{O}$ & 5,805 & 0,04 & 0,127 \\
\hline $\mathrm{N}$ & 9,26 & 0,015 & 0,054 \\
\hline $\mathrm{Al}$ & 3,449 & 0,02 & 0,038 \\
\hline $\mathrm{Si}$ & 4,149 & 0,025 & 0,045 \\
\hline $\mathrm{Cu}$ & 7,718 & 0,01 & 0,059 \\
\hline
\end{tabular}

Нейтронные дифрактограммы были получены для обеих серий образцов (после обработок ТО-1 и ТО-2) как в исходном состоянии, так и после облучения флюенсами нейтронов $1 \cdot 10^{19} \mathrm{~cm}^{-2}, 5 \cdot 10^{19} \mathrm{~cm}^{-2}, 1 \cdot 10^{20} \mathrm{~cm}^{-2}$, и дали отчетливые картины, подтверждающие присутствие в материале преципитатов.

Как видно из рис. 1, облучение быстрыми нейтронами вплоть до флюенса $10^{20} \mathrm{H} / \mathrm{cm}^{2}$ не вносит существенных изменений в нейтронограммы образцов V-4Ti-4Cr. Аналогичный вид имеют и нейтронограммы, полученные для образцов, прошедших обработку ТО-2. 


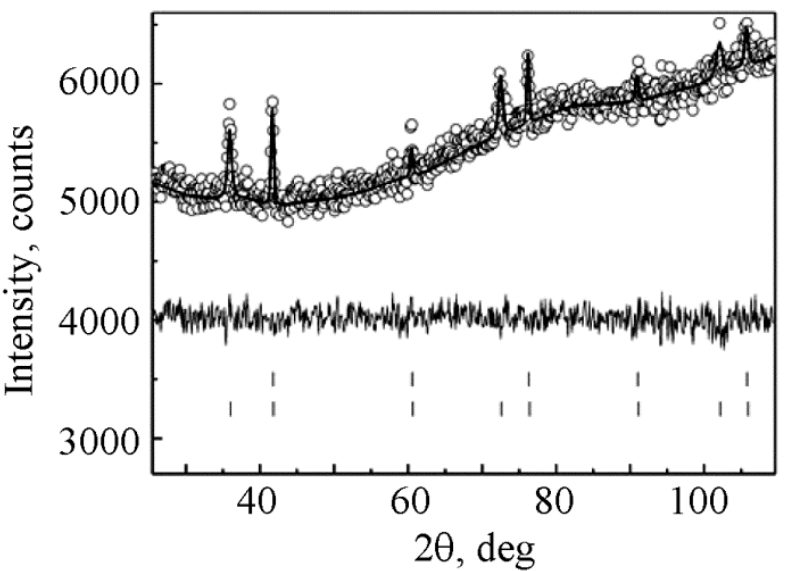

$a$

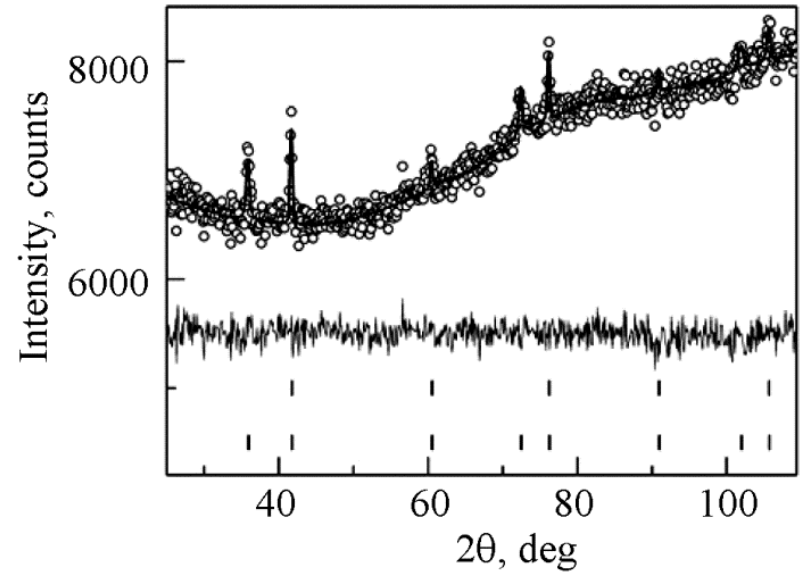

6

Рис. 1. Нейтронограммы образца V-4Ti-4Cr с обработкой TO-1 в исходном (a) и облученном (б) состояниях. Флюенс $\Phi=10^{20} \mathrm{H} / \mathrm{cm}^{2}$. Точки - эксперимент; огибающая черная линия - расчет; нижняя линия - разность между экспериментом и расчетом; верхние штрихи - угловые положения рефлексов фазы ОЦК-матрицы; нижние - оксида титана

С использованием полнопрофильного ритвелдовского анализа спектров, дополненного методикой Вильямсона-Холла, [11-13] удалось идентифицировать преципитаты окиси титана, а также оценить некоторые микроструктурные характеристики образцов.

На рис. 2 показано, как в процессе обработки спектра (образец с обработкой ТО-1, необлученный) программным пакетом FullProf происходит удаление фона и выявление дифракционных пиков ОЦК - матрицы и ТіО.

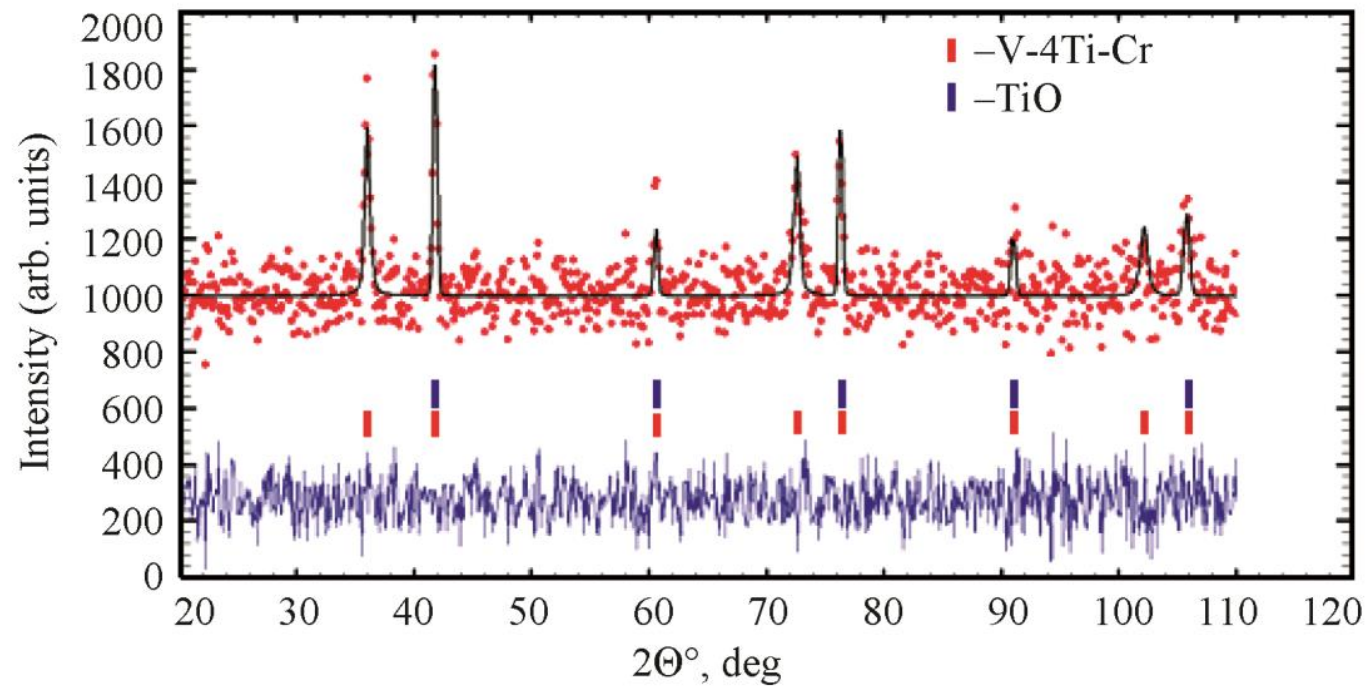

Рис. 2. Обработка нейтронограммы образца ТО-1, $\Phi=0$ программным пакетом FullProf

Отметим, что для получения пиков от почти нулевой матрицы съемки нейтронограмм пришлось проводить с большой статистикой (5 мин на точке). Для контроля на фрагментах необлученных образцов были выполнены также рентгеноструктурные исследования, подтвердившие наличие в образцах двух окристаллизованных фаз - ОЦК-матрицы и примесной TiO. Примесная фаза была идентифицирована на основе международной базы данных PDF2 (номера карточек 00-029-1361 и 01-086-2352). На рис. 3 для иллюстрации приведены с нало- 
жением нейтронный и рентгеновский дифракционные спектры образца после ТО-1, построенные для совпадения масштаба по оси абсцисс в зависимости от $x=q / 4 \pi=\sin \theta / \lambda$.

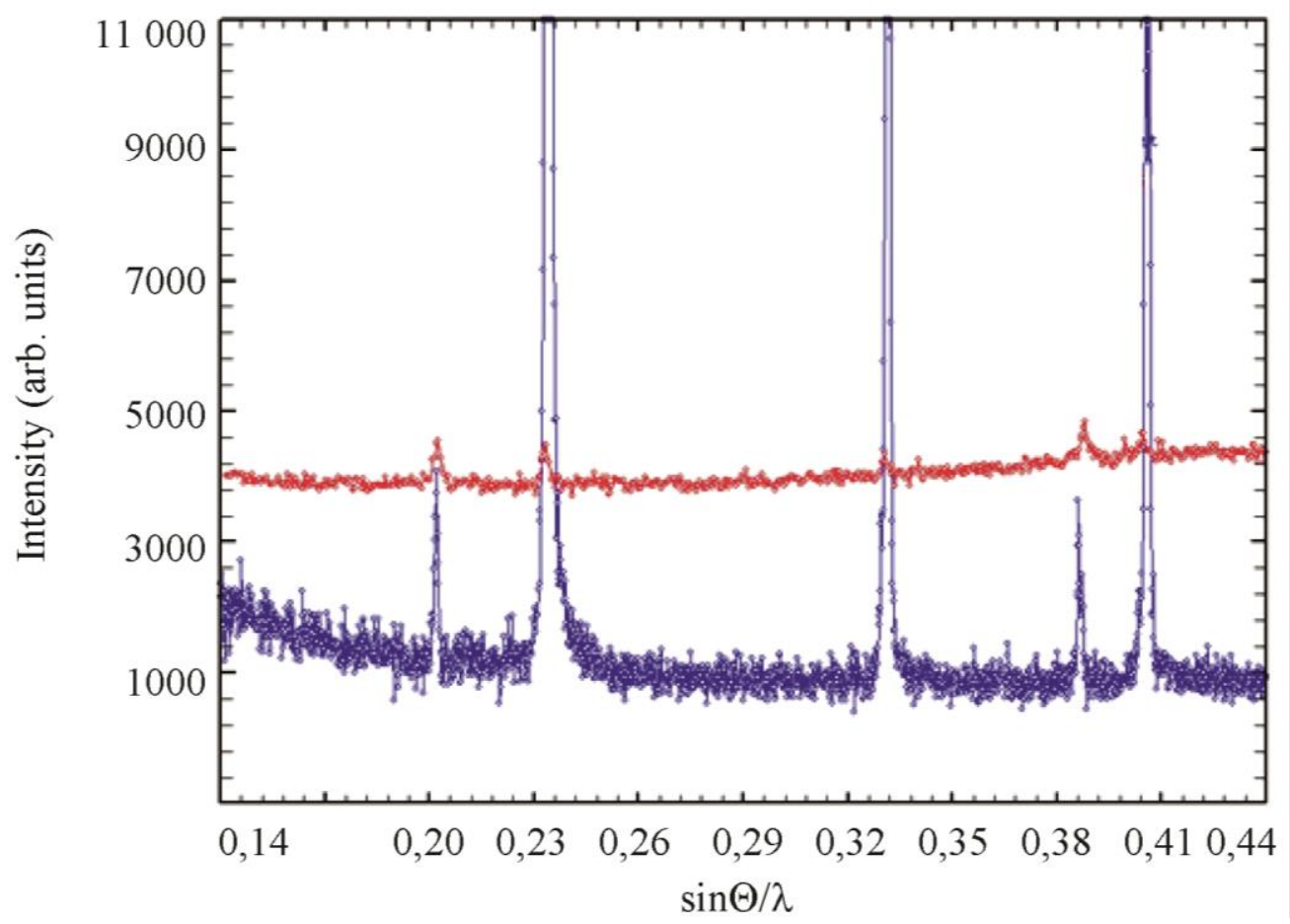

Рис. 3. Рентгеновская (синяя линия) и нейтронная (красная линия) дифрактограммы образца с ТО-1, $\Phi=0$

Эти данные явились основанием для принятия для дальнейшего анализа двухфазной модели, в ходе использования которой уширения пиков от матрицы были связаны с микронапряжениями в зернах (крупных с точки зрения дифракции), а в примесной фазе - с малыми размерами ее преципитатов. Напомним, что нейтронографические данные характеризуют объемное структурное состояние образцов в отличие от рентгеновских, показывающих состав поверхности исследованного фрагмента.

Результаты проведенного анализа представлены в табл. 3.

Таблица 3 - Микроструктурные характеристики образцов V-4Ti-4Cr, облученных разными флюенсами быстрых нейтронов

\begin{tabular}{|c|c|c|c|c|c|}
\hline Параметр & $\Phi=0$ & $\Phi=1 \cdot{ }_{2}^{10^{19}} \mathrm{~cm}$ & $\Phi=5 \cdot 10^{19} \mathrm{~cm}^{-}$ & $\Phi=1 \cdot 10_{2}^{20} \mathrm{~cm}^{-}$ & Режим \\
\hline $\mathrm{a}, \AA$ & $3,0241(5)$ & $3,0258(8)$ & $3,0260(6)$ & $3,0261(6)$ & \multirow{4}{*}{ TO-1 } \\
\hline$\Delta \mathrm{d} / \mathrm{d}, 10^{-4}$ & 0,0 & 0,0 & $3,8(6)$ & $5,5(7)$ & \\
\hline $\mathrm{TiO}$, вес. $\%$ & $0,50(5)$ & $0,47(8)$ & $0,42(8)$ & $0,35(7)$ & \\
\hline TiO: $L_{\text {окр }}, \AA$ & $247(50)$ & $306(50)$ & $261(50)$ & $241(50)$ & \\
\hline $\mathrm{a}, \AA$ & $3,0250(5)$ & $3,0265(6)$ & $3,0259(6)$ & $3,0254(5)$ & \multirow{4}{*}{ TO-2 } \\
\hline$\Delta \mathrm{d} / \mathrm{d}, 10^{-4}$ & $3,9(7)$ & 0,0 & 0,0 & 0,0 & \\
\hline TiO, вес. \% & $0,54(7)$ & $0,51(8)$ & $0,49(5)$ & $0,50(5)$ & \\
\hline TiO: $L_{\text {окр }} \AA$ & $277(50)$ & $270(50)$ & $310(50)$ & $260(50)$ & \\
\hline
\end{tabular}

Здесь $L_{\text {окр }}$ размер областей когерентного рассеяния, определенный для преципитатов $\mathrm{TiO} ; \Delta \mathrm{d} / \mathrm{d}$ - средняя (по направлениям) величина микродеформаций решетки. 
Из табл. 3 следует, что с ростом флюенса происходят небольшие изменения параметра решетки, а также уменьшение содержания оксида титана в виде преципитатов. В образцах, подготовленных в режиме ТО-2, судя по поведению микроискажений решетки, под облучением снимаются изначальные напряжения. В образцах ТО-1, наоборот, микронапряжения возникают после облучения большими флюенсами.

Эффективным методом изучения нанонеоднородностей в системах является малоугловое рассеяние нейтронов (МУРН), проведенное для вышеописанных образцов на дифрактометре D6. На рис. 4 и 5 в логарифмическом масштабе приведены зависимости сечений малоуглового рассеяния от вектора рассеяния $q(q=4 \pi \sin \theta / \lambda, \theta-$ угол рассеяния; $\lambda$ - длина волны нейтронов) для образцов, прошедших обработки ТО-1 и ТО-2 соответственно.

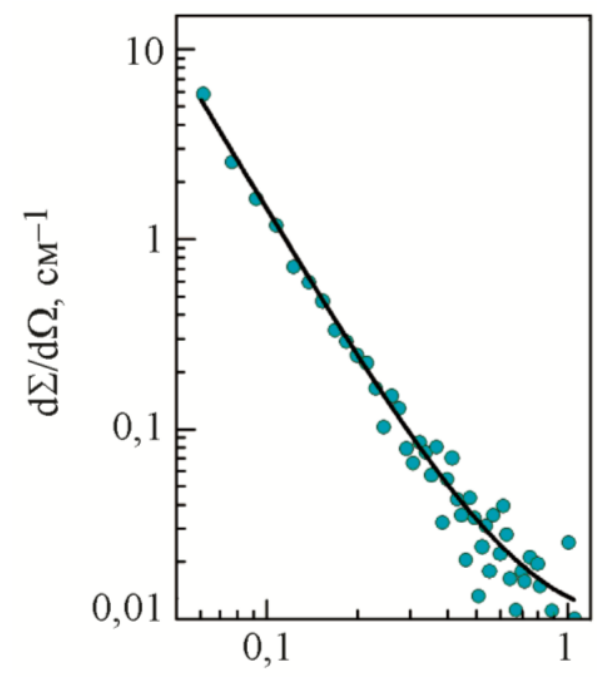

$a$

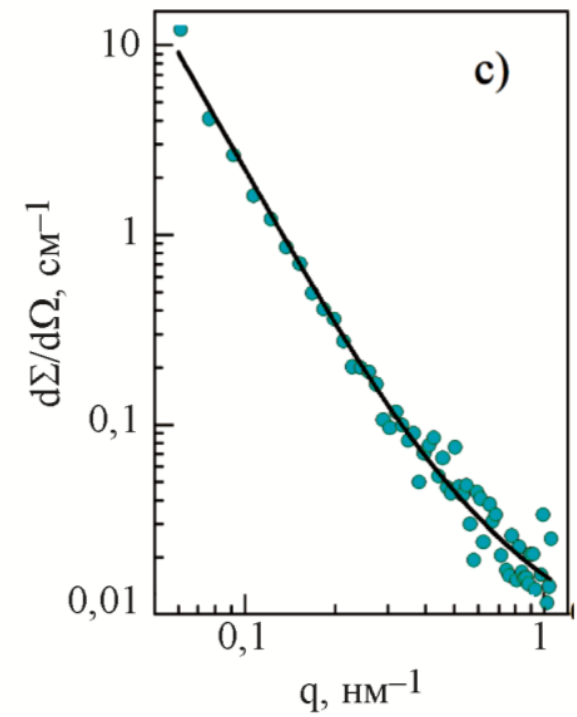

B

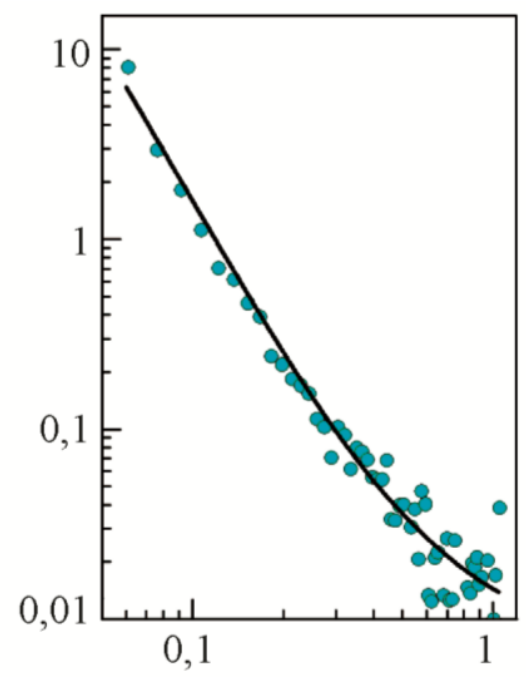

$\sigma$

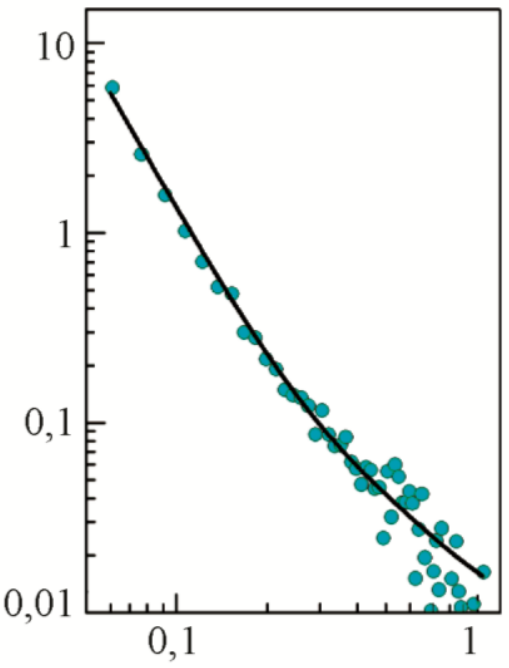

q, $\mathrm{HM}^{-1}$

2

Рис. 4. Дифрактограммы малоуглового рассеяния нейтронов на образцах сплава V-4Ti-4Cr после термообработки ТО-1 и облучения флюенсами быстрых нейтронов: $(a) \Phi=0 \mathrm{~cm}^{-2}$;

(б) $\Phi=1 \cdot 10^{19} \mathrm{~cm}^{-2} ;$ (в) $\Phi=5 \cdot 10^{19} \mathrm{~cm}^{-2}$; (2) $\Phi=1 \cdot 10^{20} \mathrm{~cm}^{-2}$. Точки - эксперимент; линии - модельный расчет 
Поведение кривых МУРН свидетельствует о наличии в системе частиц двух характерных размеров. Малые частицы дают основной вклад в правую ветвь кривой рассеяния, относительно крупные - в левую. Такое деление весьма условно, поскольку, судя по небольшой величине «излома», различие в величине размеров «малых» и «больших» частиц невелико.

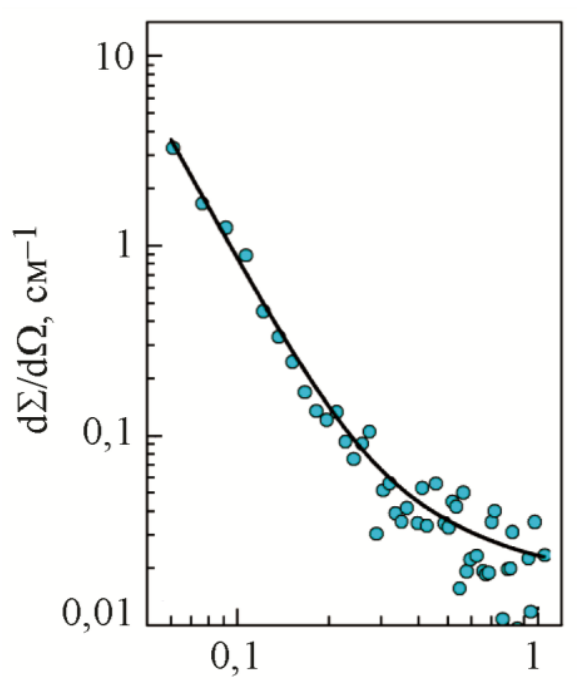

$a$

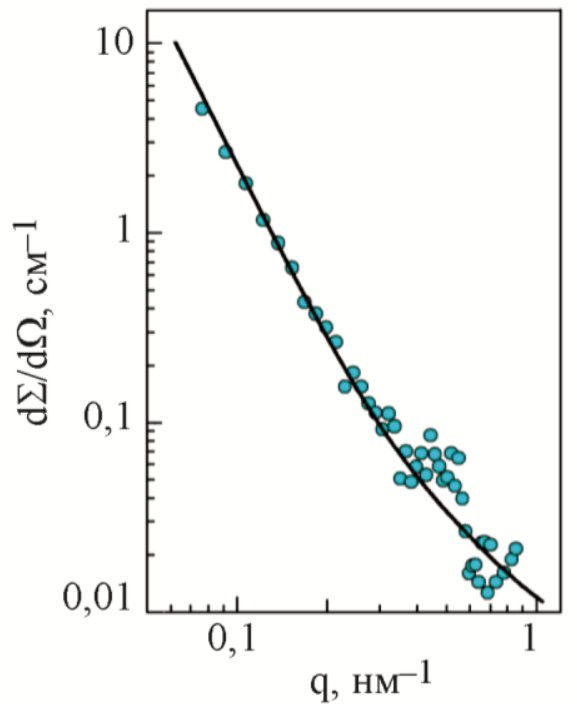

B

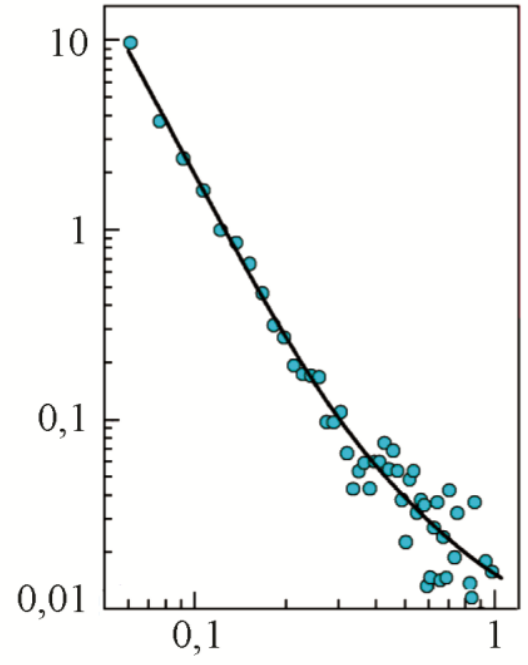

$\sigma$

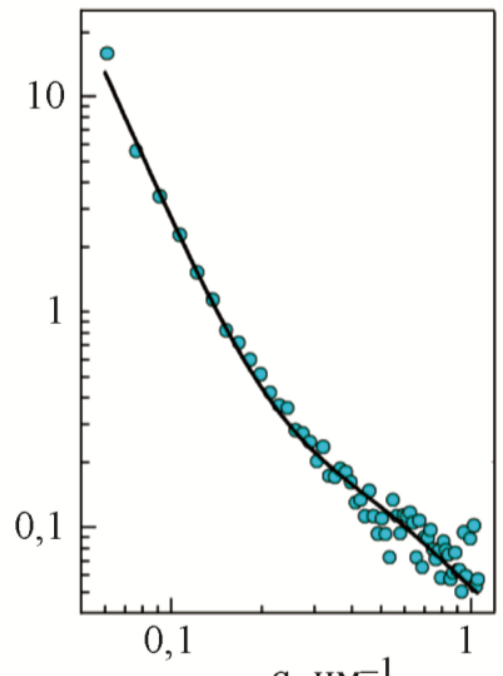

2

Рис. 5. Дифрактограммы малоуглового рассеяния нейтронов на образцах сплава V-4Ti-4Cr после термообработки ТО-2 и облучения флюенсами быстрых нейтронов: $(a) \Phi=0 \mathrm{~cm}^{-2}$; (б) $\Phi=1 \cdot 10^{19} \mathrm{~cm}^{-2} ;\left(\right.$ b) $\Phi=5 \cdot 10^{19} \mathrm{~cm}^{-2}$; (2) $\Phi=1 \cdot 10^{20} \mathrm{~cm}^{-2}$

Для описания вклада в сечение рассеяния от первых частиц (мода 1) использовалась интерполяционная формула Дебая-Порода, пригодная для случая частиц однородного размера с негладкой поверхностью $[14,15]$. Вклад от крупных частиц (мода 2) описывался в предположении их степенного распределения по размерам. 


$$
\begin{gathered}
\left(\frac{d \Sigma}{d \Omega}\right)=\left(\frac{d \Sigma}{d \Omega}\right)_{\bmod 1}+\left(\frac{d \Sigma}{d \Omega}\right)_{\bmod 2} ; \\
\left(\frac{d \Sigma}{d \Omega}\right)_{\bmod 1}=\frac{8 \pi c(1-c) \rho^{2} r^{3}}{\left(1+A q^{2} r^{2}\right)^{\frac{6-D_{S}}{2}} ;} \\
\left(\frac{d \Sigma}{d \Omega}\right)_{\bmod 2}=\left(\frac{4 \pi}{3}\right)^{3} \rho^{2} N_{2}(2+\Delta) R_{\min }^{6}\left(q R_{\min }\right)^{-4+\Delta} \int_{q R_{\min }}^{q R_{\max }} x^{-\Delta} J_{3 / 2}^{2}(x) d x .
\end{gathered}
$$

Фигурирующий в формуле (2) интерполяционный коэффициент $A$ определяется из соотношения:

$$
A^{\frac{6-D_{S}}{2}}=\frac{2}{\Gamma\left(5-D_{S}\right) \sin \left(\frac{\pi}{2}\left(D_{S}-1\right)\right)}
$$

а параметры имеют следующий смысл: $\rho$ - контраст; $r$ - радиус неоднородностей; $c$ - их объемная доля; $D_{S}-$ поверхностная фрактальная размерность; $\Gamma(x)$ - гамма-функция; $\mathrm{J}_{3 / 2}(x)-$ функция Бесселя.

При малых углах вклад (2) имеет тенденцию к насыщению, а на асимптотике (при $q \rightarrow \propto$ ) закон рассеяния принимает вид $I \sim q^{-n}$, где $n-$ так называемый параметр Порода, связанный с фрактальной размерностью $D_{\mathrm{S}}$ соотношением $n=6-D_{\mathrm{S}}$ и изменяющийся в пределах $3<n \leq 4$. Для нефрактальных объектов $n=4$.

Вклад (3) используют в случае объектов со степенным распределением рассеивающих частиц по размерам, характеризуемым параметром $\Delta$ и имеющим вид $[16,17]$ :

$$
N(R)=\left\{\begin{array}{cl}
0 ; & R<R_{\min } \\
B \cdot\left(\frac{R_{\min }}{R}\right)^{3+\Delta} ; & R_{\min } \leq R \leq R_{\max } \\
0 ; & R>R_{\max }
\end{array}\right.
$$

Величина $B$ связана с полным числом частиц $N_{2}$ в единице объема с и параметром $\Delta$ :

$$
B=\frac{N_{2}(2+\Delta)}{R_{\min }},
$$

она задается условием нормировки:

$$
\int_{0}^{\infty} N(R) d R=N_{2} .
$$

В случае МУРН под контрастом $\rho$ понимается разность плотностей амплитуд когерентного рассеяния нейтронов матрицы $\rho_{0}$ и микровключений $\rho_{i}[18]$, вычисляемых как сумма амплитуд когерентного рассеяния всех атомов элементарной ячейки рассеивателя, деленная на ее объем. Нетрудно понять, что в случае плохо окристаллизованных включений, а таковыми, судя по дифрактограммам на рис. 1-3, являются все микровключения помимо 
выявленной там фазы ТiO, о возможном составе их можно судить только руководствуясь табл. 2.

Для нашей почти нулевой матрицы оказалось, что $\rho_{0}=3,5 \cdot 10^{-10} \mathrm{~cm}^{-2}$. Что касается минорных преципитатов, то для оценки возможных значений $\rho_{i}$ были проведены вычисления этих величин для целого ряда фаз (оксидов, карбидов, нитридов, оксикарбонитридов ванадия, титана, хрома). Часть наших результатов представлена в табл. 4.

Таблица 4 - Плотность амплитуды когерентного рассеяния нейтронов различных фаз карбидов, нитридов, оксидов ванадия, титана, хрома

\begin{tabular}{|c|c|c|c|c|c|}
\hline Фаза & Структура & $\rho_{i}, 10^{10} \mathrm{~cm}^{-2}$ & Фаза & Структура & $\rho_{i}, 10^{10}$ см$^{-2}$ \\
\hline $\mathrm{TiC}$ & $\mathrm{K} 5$ (тип $\mathrm{NaCl})$ & 1,58 & $\mathrm{VO}_{2}$ & Т4 (рутил) & 1,88 \\
\hline $\mathrm{VC}$ & $\mathrm{K} 5$ (тип $\mathrm{NaCl})$ & 3,49 & $\mathrm{CrO}_{2}$ & Т4 (рутил) & 5,34 \\
\hline $\mathrm{VN}$ & $\mathrm{K} 5$ (тип $\mathrm{NaCl})$ & 5,02 & $\mathrm{~V}_{2} \mathrm{O}_{5}$ & Ромбическая & 3,16 \\
\hline $\mathrm{TiN}$ & $\mathrm{K} 5$ (тип $\mathrm{NaCl})$ & 3,05 & $\mathrm{VCrO}_{4}$ & Ромбическая & 3,85 \\
\hline $\mathrm{TiO}$ & $\mathrm{K} 5$ (тип $\mathrm{NaCl})$ & 1,24 & $\mathrm{CrO}_{3}$ & Ромбическая & 5,46 \\
\hline $\mathrm{VO}$ & $\mathrm{K} 5$ (тип $\mathrm{NaCl})$ & 3,13 & $\mathrm{Cr}_{3} \mathrm{C}_{2}$ & Ромбическая & 4,05 \\
\hline $\mathrm{CrN}$ & $\mathrm{K} 5$ (тип $\mathrm{NaCl})$ & 6,72 & $\mathrm{~V}_{2} \mathrm{C}$ & Гексагон & 3,17 \\
\hline $\mathrm{TiO}_{2}$ & $\mathrm{~T} 4$ (рутил) & 2,6 & $\mathrm{Cr}_{2} \mathrm{~N}$ & Гексагон & 10,5 \\
\hline
\end{tabular}

Как видно из таблицы, диапазон величины плотности амплитуды когерентного рассеяния нейтронов для этих фаз весьма широк: $\rho_{i}=(1-10) \times 10^{10} \mathrm{~cm}^{-2}$. Ввиду отсутствия детальных сведений о составе преципитатов при расчетах было выбрано некое среднее значение величины контраста $\rho=3,5 \times 10^{10} \mathrm{~cm}^{-2}$.

При описании экспериментальных зависимостей модельными функциями (2) и (3) подгоночными параметрами являлись величины $(r, c, n)$ и $\left(R_{\min }, N_{2}, \Delta\right)$, соответственно. Верхний предел размера в функции распределения (5) принимался равным $R_{\max }=0,5$ мкм. Ввиду малости эффекта рассеяния в больших углах и невозможности на нашем приборе проследить закон рассеяния на асимптотике фрактальная размерность $D_{\mathrm{S}}$ принималась равной 2 (параметр Порода $n=4$, что означает пренебрежение фрактальностью частиц). В условиях такого выбора была проведена подгонка модельных кривых к экспериментальным результатам (рис. 4 и 5), а по подгоночным параметрам определены следующие характеристики надатомной структуры образцов:

- объемная доля $c$ частиц моды 1 , их размер $2 r$ и объемная плотность числа частиц этой моды

$$
N_{1}=3 c / 4 \pi r^{3}
$$

- параметр $\Delta$ степенного распределения частиц моды 2, их минимальный размер $2 R_{\min }$, полная объемная плотность $N_{2}$, а по ним объемная доля $C$ и средний размер частиц $2 \bar{R}$ этой моды:

$$
\begin{gathered}
C=\frac{4 \pi}{3} \int_{0}^{\infty} R^{3} N(R) d R \\
\bar{R}=\frac{1}{N_{2}} \int_{0}^{\infty} R N(R) d R .
\end{gathered}
$$

Полученные при анализе результаты представлены в табл. 5. 
Из изложенного ясно, что результаты МУРН обрабатывались в рамках весьма упрощенной и грубой модели, а ограниченные экспериментальные возможности прибора Д6 привели к принятию дополнительных упрощающих предположений. Поэтому результаты, приведенные в табл. 5, носят оценочный, полукачественный характер.

Из рис. 4, 5 и табл. 5 видно, что ни термообработка, ни облучение быстрыми нейтронами не приводят к каким-либо качественным изменениям в картине малоуглового рассеяния нейтронов.

Таблица 5 - Параметры надатомной структуры образцов сплава V-4Ti-4Cr

\begin{tabular}{|c|c|c|c|c|c|c|c|c|c|}
\hline \multirow{2}{*}{\multicolumn{2}{|c|}{$\begin{array}{c}\text { Образец: } \\
\text { Термо- } \\
\text { обработка, } \\
\text { флюенс, см }{ }^{-2}\end{array}$}} & \multicolumn{3}{|c|}{ Мода 1} & \multicolumn{5}{|c|}{ Мода 2} \\
\hline & & $2 \boldsymbol{r}, \mathrm{HM}$ & $c, \%$ & $\begin{array}{c}N_{1}, \\
10^{23} \mathrm{M}^{-3}\end{array}$ & $\begin{array}{c}2 R_{\min }, \\
\mathrm{HM}\end{array}$ & $\begin{array}{l}2 \bar{R}, \\
\mathrm{HM}\end{array}$ & $\begin{array}{c}N_{2}, \\
10^{21} \mathrm{M}^{-3}\end{array}$ & $\Delta$ & $C, \%$ \\
\hline \multirow{4}{*}{ TO-1 } & 0 & 1,8 & 0,025 & 0,8 & 3,6 & 5,1 & 1,7 & 1,35 & 0,03 \\
\hline & $1 \cdot 10^{19}$ & 2,0 & 0,05 & 1,2 & 3,6 & 5,1 & 1,6 & 1,25 & 0,032 \\
\hline & $5 \cdot 10^{19}$ & 2,0 & 0,07 & 1,7 & 3,6 & 5,2 & 2,0 & 1,2 & 0,043 \\
\hline & $1 \cdot 10^{20}$ & 2,2 & 0,09 & 1,6 & 3,6 & 5,2 & 1,2 & 1,2 & 0,026 \\
\hline \multirow{4}{*}{ TO-2 } & 0 & 2,0 & 0,05 & 1,2 & 3,6 & 5,3 & 0,6 & 1,1 & 0,015 \\
\hline & $1 \cdot 10^{19}$ & 2,2 & 0,07 & 1,3 & 3,6 & 5,3 & 1,1 & 1,0 & 0,033 \\
\hline & $5 \cdot 10^{19}$ & 2,2 & 0,07 & 1,3 & 3,6 & 5,6 & 0,8 & 0,8 & 0,034 \\
\hline & $1 \cdot 10^{20}$ & 2,0 & 0,5 & 11,2 & 3,6 & 5,5 & 0,9 & 0,8 & 0,038 \\
\hline
\end{tabular}

Характеристики больших частиц (мода 2) почти не зависят от типа термообработки и нейтронного облучения. Минимальный размер больших частиц составляет 3,2 нм, а средний размер находится в пределах $(5,1-5,6)$ нм. Плотность и объемная доля больших частиц слабо меняются нерегулярным образом и находятся в пределах $N_{2}=(0,6-2) \cdot 10^{21} \mathrm{M}^{-3}$ и $C=(0,02-$ $0,04) \%$. Вместе с тем, параметр $\Delta$ с флюенсом уменьшается примерно на $20 \%$. Это свидетельствует о том, что «хвосты» кривой степенного распределения (5) с ростом флюенса становятся более затянутыми. (Напомним, что выявленные ранее в ходе нейтроннодифракционных исследований хорошо окристаллизованные преципитаты ТiO имели размеры порядка 30 нм и не дают вклад в наблюдавшийся нами спектр МУРН.)

Из подгоночных расчетов следует, что размер малых частиц (мода 1) составляет около 2 нм и почти не зависит от термообработки и воздействия быстрых нейтронов. Для таких частиц после обработки ТО-1 численная плотность $N_{1}$ по порядку величины составляет $10^{23} \mathrm{M}^{-3}$, а объемная доля - сотые доли процента. С ростом флюенса до $1 \cdot 10^{20} \mathrm{~cm}^{-1}$ происходит двукратное монотонное увеличение $N_{1}$. Поскольку при этом расчет дает также увеличение линейных размеров частиц, то их объемная доля возрастает в 3,6 раза. Примерно такая же картина наблюдалась для малых частиц и после обработки ТО-2 при облучении до флюенса $5 \cdot 10^{19} \mathrm{~cm}^{-1}$. Однако обращает на себя внимание выявленное существенное изменение числа $N_{1}$ и концентрации $c$ после облучения флюенсом $1 \cdot 10^{20} \mathrm{~cm}^{-1}$, хорошо заметное также по сдвигу шкалы отсчета на оси ординат рисунка $5 \mathrm{~d}$ т. е по явному, экспериментально наблюдаемому усилению интенсивности малоуглового рассеяния.

Отметим, что вид кривой рассеяния определяется в основном большими неоднородностями. Вклад малых частиц проявляется в правой части кривой рассеяния при $q>(0,3-0,4) \mathrm{Hм}^{-1}$. По этой причине погрешность определения параметров малых частиц оказывается большой. Можно утверждать лишь, что размер малых частиц составляет 1-2 нм. Весьма грубым является предположение об одинаковой величине контраста $\rho$ для всех частиц. Как нетрудно ви- 
деть из табл. 4, ошибка тут при аддитивном учете в (2) разных фракций может составлять и $100 \%$, и $200 \%$.

\section{4. Заключение}

Выполненное исследование сплава V-4Ti-4Cr методами нейтронной дифракции и малоуглового рассеяния нейтронов показало применимость и перспективность этих методов для изучения радиационных дефектов даже в таких, не очень удобных с точки зрения нейтронографии, материалах. В ходе исследования удалось определить характеристики микроструктуры сплава, сформированной преципитатами разных размеров, и изучить их радиационно-индуцированные изменения.

Более глубокое исследование обнаруженных с помощью малоуглового рассеяния нейтронов эффектов требует усложнения привлекаемых для анализа экспериментальных данных моделей с привлечением результатов других методик для уточнения их параметров, а также кардинального улучшения характеристик дифрактометра МУРН.

В исследованном диапазоне доз ни термообработка, ни нейтронное облучение не вызвали существенных изменений спектров нейтронной дифракции и малоуглового рассеяния. Очевидно, представляет большой интерес проведение аналогичных исследований данного материала в условиях высокодозного (измеряющегося десятками СНА - смещений на атом) нейтронного облучения.

\section{Благодарность}

Работа выполнена с использованием УНУ «НМК ИФМ» в рамках государственного задания МИНОБРНАУКИ России (тема «Поток» № АААА-А18-118020190112-8).

\section{Литература}

1. IAEA activities on coordinated research of structural materials for advanced reactor systems / A. Zeman, R. Kaiser, V. Inozemtsev, R. L. Beatty // J. Nucl. Mater. - 2012. - Vol. 428. - P. 3-5. DOI: 10.1016/j.jnucmat.2012.06.024.

2. Was G. S. Fundamentals of Radiation Materials Science. Metals and Alloys. - New York : Springer Verlag, 2007. - 1002 p. - ISBN 978-1-4939-3436-2. - DOI: 10.1007/978-1-4939-3438-6.

3 . Neutron diffraction study of structure and phase composition of fuel claddings made of colddeformed steel ChS68 after normal operation in BN-600 reactor / V. I. Voronin, I. F. Berger, N. V. Proskurnina, B. N. Goshchitskii // J. Nucl. Mater. - 2018. - Vol. 509. - P. 218-224. DOI: $10.1016 /$ j.jnucmat.2018.06.036.

4. Voronin V. I. Neutronography of Irradiated Reactor Austenitic Steels // Crystallography Reports. - 2021. - Vol. 66, No. 2. - P. 314-322. - DOI: 10.1134/S1063774521020127.

5. Peculiarities of radiation-induced processes in the $\mathrm{Cr}-\mathrm{Ni}$-Mo austenitic steels studied by neutron diffraction / V. I. Voronin, V. L. Arbuzov, V. I. Bobrovskii, S. E. Danilov, K. A. Kozlov, N. V. Proskurnina, V. V. Sagaradze // Diagnostics, Resource and Mechanics of materials and structures. - 2015. - Iss. 5. - P. 80-89. - DOI: 10.17804/2410-9908.2015.5.080-089. URL: DREAM_Issue_5_2015_Voronin V.I. et al._080_089.docx

6. Influence of the austenitic stainless steel microstructure on the void swelling under ion irradiation / B. Rouxel, C. Bisor, Y. De Carlan, A. Courcelle, A. Legris // EPJ Nuclear Sci. Technol. 2016. - Vol. 2. - P. 30. - DOI: 10.1051/epjn/2016023.

7. The accumulation and annealing of radiation-induced defects and effect of hydrogen on the physicomechanical properties of the $\mathrm{V}-4 \mathrm{Ti}-4 \mathrm{Cr}$ vanadium-based alloys under low-temperature (at 77 K) neutron irradiation / V. L. Arbuzov, B. N. Goshchitskii, V. V. Sagaradze, S. E. Danilov, Yu. N. Zuev, A. E. Kar'kin, V. D. Parkhomenko, A. V. Kozlov, V. M. Chernov // Physics of Metals and Metallography. - 2016. - Vol. 117, No. 3. - P. 299-306. - DOI: 10.1134/S0031918X16030030. 
8. Микроструктура сплава V-4Ti-4Cr, облученного электронами, нейтронами и ионами дейтерия после различных термообработок / В. Л. Арбузов, Б. Н. Гощицкий, С. Е. Данилов, В. В. Сагарадзе, О. В. Антонова, Г. А. Распопова, В. М. Чернов, А. В. Козлов // Вопросы атомной науки и техники. Сер. Материаловедение и новые материалы. - 2018. - № 3 (94). C. 4-12.

9. Nuclear Physical Properties of Ferritic-Martensitic Steel EK-181 under Conditions of Long Term Neutron Irradiation in Fast Breeder BN-600 and Fusion DEMO-RF Reactors / D. A. Blokhin, M. V. Leontyeva-Smirnova, V. M. Chernov, A. I. Blokhin, N. A. Demin, I. V. Sipachev // Inorganic Materials: Applied Research. - 2011. - Vol. 2, No. 2. - P. 129-135. DOI: $10.1134 / \mathrm{S} 2075113311020067$.

10. Structure-Phase Transformations and Physical Properties of Ferritic-Martensitic $12 \%$ Chromium Steels EK-181 and ChS-139 / V. M. Chernov, M. V. Leont'eva-Smirnova, M. M. Potapenko, N. A. Polekhina, I. Yu. Litovchenko, A. N. Tyumentsev, E. G. Astafurova, and L. P. Khromova // Technical Physics. - 2016. - Vol. 61, No. 1. - P. 97-102. DOI: $10.1134 / \mathrm{S} 1063784216010084$.

11. Rodriguez-Carvajal J. Recent advances in magnetic structure determination by neutron powder diffraction // Physica B. - 1993. - Vol. 192. - P. 55-64. - DOI: 10.1016/09214526(93)90108-I.

12. Ungar T., Borbely A. The effect of dislocation contrast on x-ray line broadening: a new approach to line profile analysis // Appl. Phys. Lett. - 1996. - Vol. 69 (21). - P. 3173-3175. DOI: $10.1063 / 1.117951$.

13. Dislocation structure evolution induced by irradiation and plastic deformation in the $\mathrm{Zr}-2.5 \mathrm{Nb}$ nuclear structural material determined by neutron diffraction line profile analysis / L. Balogh, D. W. Brown, P. Mosbrucker, F. Long, M. R. Daymond // Acta Materialia. - 2012. Vol. 60. - P. 5567-5577. - DOI:10.1016/j.actamat.2012.06.062.

14. Bale H. D., Schmidt P. W. Small angle X-ray scattering investigation of submicroscopic porosity with fractal properties // Phys. Rev. Lett. - 1984. - Vol. 53. - P. 596-604. DOI: 10.1103/physrevlett.53.596.

15. Debye P., Anderson H. R., Brumberger H. Scattering by an inhomogeneous solid. II. The correlation function and its application // J. Apll. Phys. - 1957. - Vol. 28. - P. 679-684.

16. Small-Angle Neutron Scattering Investigation of the Nanostructure of Ferritic- Martensitic $12 \%$-Chromium Steels / S. G. Bogdanov, B. N. Goshchitskii, V. D. Parkhomenko, M. V. Leontieva-Smirnova, V. M. Chernov // Physics of Solid State. - 2014. - Vol. 56, No. 1. P. 1-13. - DOI: 10.1134/S1063783414010065.

17. Small-Angle Neutron Scattering Investigation of the Substructure of Nickel Irradiated with Fast Neutrons / S. G. Bogdanov, B. N. Goshchitskii, V. D. Parkhomenko // Physics of Metals and Metallography. - 2014. - Vol. 115, No. 6. - P. 566-569. - DOI: 10.1134/S0031918X14060039.

18. Feigin L. A., Svergun D. I. Structure Analysis by Small-Angle X-Ray and Neutron Scattering // New-York : Plenum Press, 1987. - 335 p. - ISBN 0-306-42629-3. 